\title{
Cyclicity of coefficient multipliers: Linear structure
}

\author{
L. Bernal-González, M.C. Calderón-Moreno * and J.A. Prado-Bassas
}

\begin{abstract}
In this paper we characterize various kinds of cyclicity of sequences of coefficient multipliers, which are operators defined on spaces of holomorphic functions. In the case of a single coefficient multiplier we characterize its cyclicity, which contrasts with the fact that such operators are never supercyclic. Moreover, it is proved that for each cyclic function there is a dense part of the linear span of its orbit all of whose vectors are cyclic.
\end{abstract}

\section{Introduction}

In this paper we are concerned with the various kinds of cyclicity of certain operators or sequences of operators defined on spaces of holomorphic functions. Let us first recall some basic terminology, which is standard in the setting of linear dynamical systems.

By $\mathbb{N}, \mathbb{N}_{0}, \mathbb{R}, \mathbb{C}$ we denote the set of positive integers, the set $\mathbb{N} \cup\{0\}$, the real line and the complex plane, respectively. If $X$ is a (Hausdorff) topological vector space over $\mathbb{R}$ or $\mathbb{C}$, then an operator on $X$ is a continuous linear selfmapping of $X$. If $A \subset X$ then $\operatorname{span}(A)$ will stand for the linear

*Corresponding author.

The authors have been partially supported by MCYT Grants BFM2003-03893-C02-01, MTM2004-21420-E and Plan Andaluz de Investigación Junta de Andalucía FQM-127.

Key words and phrases: cyclicity, supercyclicity, hypercyclicity, coefficient multiplier, Euler differential operator, Hadamard operator.

2000 Mathematics Subject Classification: Primary 47A16. Secondary 30E10, 30B10. 
span of $A$. We say that a sequence $\left(T_{n}\right)_{n}$ of operators on $X$ is hypercyclic if there exists a vector $x \in X$-called hypercyclic for $\left(T_{n}\right)_{n}-$ such that its orbit $\operatorname{Orb}\left(\left(T_{n}\right), x\right):=\left\{T_{n} x: n \in \mathbb{N}\right\}$ is dense in $X$. If we replace the orbit of the vector $x$ to its projective orbit $\left\{\lambda T_{n} x: n \in \mathbb{N}, \lambda \in \mathbb{K}\right\}(\mathbb{K}=\mathbb{R}$ or $\mathbb{C})$ or to $\operatorname{span}\left(\operatorname{Orb}\left(\left(T_{n}\right), x\right)\right)$, respectively, we obtain the (weaker) notions of supercyclicity and cyclicity. The set of cyclic (supercyclic, hypercyclic) vectors for $\left(T_{n}\right)_{n}$ will be denoted by $C\left(\left(T_{n}\right)\right)\left(S C\left(\left(T_{n}\right)\right), H C\left(\left(T_{n}\right)\right)\right.$, respectively). If now we have a single operator $T$, then we say that $T$ is cyclic (supercyclic, hypercyclic) whenever the sequence of its iterates $\left(T^{n}\right)_{n \geq 0}$ is cyclic (supercyclic, hypercyclic, resp.). Here $T^{0}=I=$ the identity on $X, T^{1}=T, T^{2}=T \circ T$ and so on.

Recall that an F-space is a complete metrizable topological vector space. For instance, the space $H(G)$ of holomorphic functions on a domain (= nonempty connected open subset) $G$ of $\mathbb{C}$, endowed with the compact-open topology, is an F-space.

The diverse kinds of cyclicity considered above have been extensively studied during the last decades for the shift-type operators (weighted, nonweighted, forward, backward, bilateral) on spaces of holomorphic functions and on diverse sequences spaces, see for instance [22], [24], [9], [18], [3], [11], [12], [17], [19], among others. Nevertheless, as far as we know, no such study on spaces of holomorphic functions has been performed yet for operators in which a sequence of weights is applied on the coefficients without shifting them.

The purpose of this paper is to contribute to filling in the last gap. The adequate operators -the coefficient multipliers- will be defined in Section 2. In Section 3, the cyclic (supercyclic, hypercyclic) sequences of coefficient multipliers are characterized, as well as the cyclicity of a single such operator. It is also shown that no coefficient multiplier is supercyclic, and that a sequence of such operators never satisfies the so-called Hypercyclicity Criterion, in spite of the fact that hypercyclic sequences of coefficient multipliers do exist. Finally, in Section 4 it is shed light on the linear structure of the set of cyclic functions; namely, we demonstrate that for each cyclic function there is a dense part of the linear span of its orbit all of whose vectors are also cyclic. 


\section{Coefficient multipliers}

Let $G$ be a domain of $\mathbb{C}$ with $0 \in G$. Assume that $X$ is a topological vector space of holomorphic functions on $G$ (so $X \subset H(G)$ ). We say that an operator $T: X \rightarrow X$ is a coefficient multiplier if there exists a sequence $\sigma=\left(a_{k}\right)_{k \geq 0}$ in $\mathbb{C}$ such that for any $f \in X$ with $f(z)=\sum_{k=0}^{\infty} f_{k} z^{k}$ around the origin, we have

$$
T f(z)=\sum_{k=0}^{\infty} a_{k} f_{k} z^{k} \quad \text { around the origin. }
$$

If $T$ is as above then we will denote $T=T_{\sigma}$. Hence $T$ acts like a "diagonal" operator.

Examples of this kind of operators can be found in the literature. Let us collect some of them.

1. If $G=\mathbb{C}(G=\{z \in \mathbb{C}:|z|<R\}$ with $0<R<\infty)$ then any sequence $\left(a_{k}\right)_{k \geq 0}$ in $\mathbb{C}$ such that $\lim \sup _{k \rightarrow \infty}\left|a_{k}\right|^{1 / k}<\infty\left(\lim \sup _{k \rightarrow \infty}\left|a_{k}\right|^{1 / k} \leq 1\right.$, resp. $)$ defines a coefficient multiplier on $H(G)$.

2. For $\alpha \in \mathbb{C}$ and sets $A, B \subset \mathbb{C}$ we denote $\alpha A=\{\alpha a: a \in A\}, A \cdot B=\{a b$ : $a \in A, b \in B\}$ and $A \odot B=\left(A^{c} \cdot B^{c}\right)^{c}$. Let $G_{1}, G_{2}$ be domains in $\mathbb{C}$ with $0 \in G_{1} \cap G_{2}$. Then for any function $g \in H\left(G_{1}\right)$ with $g(z)=\sum_{k=0}^{\infty} g_{k} z^{k}$, we can consider the Hadamard product operator $H_{g}: H\left(G_{2}\right) \rightarrow H\left(G_{1} \odot G_{2}\right)$ defined as follows. If $f \in H\left(G_{2}\right)$ and $f(z)=\sum_{k=0}^{\infty} f_{k} z^{k}$ around the origin then $H_{g} f(z)=\sum_{k=0}^{\infty} g_{k} f_{k} z^{k}$ around the origin. We have that $H_{g}$ is well-defined, continuous and linear (see [21, Theorem H]). In particular, if $G_{1}=\mathbb{C} \backslash\{1\}$ and $G_{2}=G$ is any domain with $0 \in G$ we have that $G_{1} \odot G_{2}=G$ (see [5]) and $H_{g}$ becomes a coefficient multiplier operator on $H(G)$.

3. Let $\mathcal{D}$ be the operator on $H(G)$ given by $\mathcal{D} f(z)=z f^{\prime}(z)$ and set $\mathcal{D}^{n}=$ $\mathcal{D} \circ \cdots \circ \mathcal{D}(n$-fold $)$, where $\mathcal{D}^{0}$ denotes the identity operator. For each entire function $\Phi(z)=\sum_{n \geq 0} \phi_{n} z^{n}$ of subexponential type -that is, satisfying that for every $\varepsilon>0$ there is a constant $K=K(\varepsilon) \in(0,+\infty)$ such that $|\Phi(z)| \leq$ $K e^{\varepsilon|z|}(z \in \mathbb{C})-$, we have that $\Phi$ induces an operator $\Phi(\mathcal{D})$ on $H(G)$, called the Euler differential operator, by $\Phi(\mathcal{D}) f:=\sum_{n \geq 0} \phi_{n} \mathcal{D}^{n} f$. Moreover, if $f(z)=$ $\sum_{n \geq 0} f_{n} z^{n}$ around the origin, then

$$
\Phi(\mathcal{D}) f(z)=\sum_{n \geq 0} \Phi(n) f_{n} z^{n} \text { around the origin. }
$$


Hence $\Phi(\mathcal{D})$ is a coefficient multiplier on $H(G)$. See [16, pages 46-54] and [8] for properties about the Euler differential operator.

4. Let $\mathbb{D}:=\{z \in \mathbb{C}:|z|<1\}$ and $\overline{\mathbb{D}}:=\{z \in \mathbb{C}:|z| \leq 1\}$ be the open unit disk and the closed unit disk, respectively. Let $S_{\nu}(\nu \in \mathbb{R})$ denote the Hilbert space of functions $f(z)=\sum_{n=0}^{\infty} a_{n} z^{n} \in H(\mathbb{D})$ for which the norm $\|f\|_{\nu}=\left(\sum_{n=0}^{\infty}\left|a_{n}\right|^{2}(n+1)^{2 \nu}\right)^{1 / 2}$ is finite. Observe that for $\nu=-1 / 2,0,1 / 2$ the space $S_{\nu}$ is, respectively, the classical Bergman space $B^{2}$, the Hardy space $H^{2}$, the Dirichlet space $D$ (see for instance [25] for the fundamentals on these spaces). Consider also the space $A^{\infty}:=\left\{f \in H(\mathbb{D}): f^{(n)}\right.$ has continuous extension on $\overline{\mathbb{D}}$ for all $\left.n \in \mathbb{N}_{0}\right\}$, which is an F-space when endowed with the topology of the uniform convergence on $\overline{\mathbb{D}}$ for all derivatives. By using the Cauchy estimates it is easy to see that $A^{\infty}=\left\{f(z)=\sum_{n=0}^{\infty} a_{n} z^{n} \in H(\mathbb{D})\right.$ : $\left(n^{N}\left|a_{n}\right|\right)_{n \geq 0}$ is bounded for every $\left.N \in \mathbb{N}\right\}$. If $\sigma=\left(a_{k}\right)_{k \geq 0}$ is a bounded sequence then from the definition of $S_{\nu}$ and the second expression of $A^{\infty}$ we obtain easily that the coefficient multiplier $T_{\sigma}$ is a well-defined operator on both $S_{\nu}$ and $A^{\infty}$. For purposes which will appear clearer later, we point out that the polynomials are dense in each $S_{\nu}\left(A^{\infty}\right)$ and that the topology on $S_{\nu}$ $\left(A^{\infty}\right.$, resp.) is finer than that of locally uniform convergence in $\mathbb{D}$.

5. Also the Hardy spaces $H^{p}$, the Bergman spaces $B^{p}(0<p<+\infty)$ and the spaces $A^{N}:=\left\{f \in H(\mathbb{D}): f^{(n)}\right.$ has continuous extension on $\overline{\mathbb{D}}$ for $n=0,1, \ldots, N\}\left(N \in \mathbb{N}_{0}\right)$, endowed with their respective natural distances, are F-spaces that satisfy the last two properties (see [1] and [7]), but in this case we do not know a good class of sequences $\sigma$ such that $T_{\sigma}$ is well-defined on them. Of course, if $\sigma=\left(a_{k}\right)_{k \geq 0}$ satisfies $a_{m}=a_{m+1}=a_{m+2}=\cdots$ for some $m$, then $T_{\sigma}$ is an operator on all above spaces.

\section{Cyclicity of coefficient multipliers}

From now on, we will denote by $G$ a domain in $\mathbb{C}$ with $0 \in G$, and by $X$ a topological vector space of holomorphic functions on $G$. We represent by $\left(T_{n}\right)$ a sequence of coefficient multipliers $T_{n}=T_{\sigma(n)}(n \in \mathbb{N})$ on $X$. Therefore $\sigma(n)=\left(a_{k, n}\right)_{k \geq 0}$ for each $n \in \mathbb{N}$, where the values $a_{k, n}$ are complex numbers. Hence, if $f \in X$ and $f(z)=\sum_{k=0}^{\infty} f_{k} z^{k}$ around the origin, we have

$$
T_{n} f(z)=\sum_{k=0}^{\infty} a_{k, n} f_{k} z^{k}
$$


around the origin.

Let us denote by $\mathcal{P}$ the class of polynomials with complex coefficients. In order to isolate the adequate spaces of holomorphic functions, we introduce the following concept.

Definition 3.1. Suppose that $X$ is an F-space of holomorphic functions on a domain $G$ of $\mathbb{C}$. We say that $X$ is a $C P$-space on $G$ whenever the next properties hold:

(i) Convergence in $X$ implies uniform convergence on compacta in $G$; in other words, the inclusion $X \subset H(G)$ is continuous.

(ii) The set $\mathcal{P}$ is a dense subset of $X$.

For instance, if $G$ is simply connected then due to Runge's approximation theorem the space $H(G)$ is a CP-space. Also the spaces $S_{\nu}(\nu \in \mathbb{R}), H^{p}, B^{p}$ $(0<p<+\infty)$ and $A^{N}\left(N \in \mathbb{N}_{0} \cup\{\infty\}\right)$ are CP-spaces. Of course, if $G$ is any domain of $\mathbb{C}$, the closure of $\mathcal{P}$ in $H(G)$ is a CP-space too.

In the next theorem we will characterize the cyclicity of a sequence $\left(T_{n}\right)$ as defined by (1). From now on, the set $\mathbb{C}^{\mathbb{N}_{0}}$ will be endowed with the product topology. Recall that a subset $A$ of a Baire topological space $Y$ is called residual whenever its complement is of first category; so such a subset $A$ is "very large" in $Y$.

Theorem 3.2. Let $G$ be a domain of $\mathbb{C}$ with $0 \in G$. Assume that $X$ is a $C P$-space on $G$ and that $\left(T_{n}\right)$ is a sequence of coefficient multipliers on $X$ with associated sequences $\left(a_{k, n}\right)_{k \geq 0}(n \in \mathbb{N})$. Then the following conditions are equivalent:

(a) The set $C\left(\left(T_{n}\right)\right)$ is residual in $X$.

(b) The sequence $\left(T_{n}\right)$ is cyclic.

(c) The span of $\left\{\left(a_{k, n}\right)_{k \geq 0}: n \in \mathbb{N}\right\}$ is dense in $\mathbb{C}^{\mathbb{N}_{0}}$.

Proof. It is trivial that (a) implies (b). Assume that (b) is satisfied, that is, there exists a function $f \in C\left(\left(T_{n}\right)\right)$. Then $f(z)=\sum_{k=0}^{\infty} f_{k} z^{k}$ around 0 with $f_{k} \neq 0$ for all $k \in \mathbb{N}_{0}$. Indeed, condition (i) of Definition 3.1 together with the Weierstrass convergence theorem yield that each projection $\Pi_{N}$ : $\sum g_{k} z^{k} \in X \mapsto\left(g_{0}, \ldots, g_{N}\right) \in \mathbb{C}^{N+1}\left(N \in \mathbb{N}_{0}\right)$ is continuous. But due to 
condition (ii) we obtain that $\Pi_{N}$ is also onto. In particular, each projection $P_{N}: \sum g_{k} z^{k} \in X \mapsto g_{N} \in \mathbb{C}$ is also continuous and onto, so the cyclicity of $f$ forces to $f_{N} \neq 0\left(N \in \mathbb{N}_{0}\right)$. Now, the set $\left\{P\left(T_{n}\right) f: n \in \mathbb{N}, P \in \mathcal{P}\right\}$ is dense in $X$. Consequently, $\Pi_{N}\left(\left\{P\left(T_{n}\right) f: n \in \mathbb{N}, P \in \mathcal{P}\right\}\right)$ must be dense in $\mathbb{C}^{N+1}$. This implies that the span of $\left\{\left(a_{k, n} f_{k}\right)_{k>0}: n \in \mathbb{N}\right\}$ is dense in $\mathbb{C}^{\mathbb{N}_{0}}$. But the last span is the same as the span of $\left\{\left(a_{k, n}\right)_{k \geq 0}: n \in \mathbb{N}\right\}$, because $f_{k} \neq 0$ for all $k$. Hence (c) has been proved.

It remains only to show that (c) implies (a). Observe that $X$ is a secondcountable Baire space, because it is an F-space and $\mathcal{P}$ is a dense subset of it. Therefore by [10, Theorem 1] (as applied to $Y=X$ and to the family of operators $\left.\left\{\sum_{n=1}^{N} \lambda_{n} T_{n}: N \in \mathbb{N} ; \lambda_{1}, \ldots, \lambda_{N} \in \mathbb{C}\right\}\right)$, we have to see that the set

$$
S:=\left\{\left(f, \sum_{n=1}^{N} \lambda_{n} T_{n} f\right): N \in \mathbb{N} ; \lambda_{1}, \ldots, \lambda_{N} \in \mathbb{C} ; f \in X\right\}
$$

is dense in $X \times X$.

Fix two polynomials $p(z)=\sum_{k=0}^{\alpha} p_{k} z^{k}, q(z)=\sum_{k=0}^{\beta} q_{k} z^{k}$ (so $p, q \in X$ ) with $p_{k} \neq 0$ for all $k \in\{0, \ldots, \alpha\}$. We can suppose with no loss of generality that $\alpha<\beta$.

By hypothesis, there exist a sequence $\left(N_{j}\right)_{j} \subset \mathbb{N}$ and finite sequences $\left(\lambda_{j, n}\right)_{n=1}^{N_{j}}(j \in \mathbb{N})$ of complex numbers such that

$$
\sum_{n=1}^{N_{j}} \lambda_{j, n} a_{k, n} \rightarrow\left\{\begin{array}{ll}
q_{k} / p_{k} & \text { if } 0 \leq k \leq \alpha \\
\infty & \text { if } \alpha+1 \leq k \leq \beta
\end{array} \quad(j \rightarrow \infty) .\right.
$$

Observe that we can assume with no loss of generality that no sum $s(j, k):=$ $\sum_{n=1}^{N_{j}} \lambda_{j, n} a_{k, n}(j \in \mathbb{N}, k \in\{0, \ldots, \beta\})$ is zero. For each $j \in \mathbb{N}$ and $k \in$ $\{0,1, \ldots, \beta\}$ we define

$$
f_{k, j}=\left\{\begin{array}{cl}
p_{k} & \text { if } 0 \leq k \leq \alpha \\
q_{k} / s(j, k) & \text { if } \quad \alpha+1 \leq k \leq \beta .
\end{array}\right.
$$

Hence, by (3),

$$
f_{k, j} \rightarrow\left\{\begin{array}{ll}
p_{k} & \text { if } 0 \leq k \leq \alpha \\
0 & \text { if } \quad \alpha+1 \leq k \leq \beta
\end{array} \quad(j \rightarrow \infty)\right.
$$

and

$$
s(j, k) f_{k, j} \rightarrow q_{k} \quad(j \rightarrow \infty) \quad \text { for all } k=0, \ldots, \beta \text {. }
$$


For each $j \in \mathbb{N}$ we consider the polynomial $f_{j}$ given by $f_{j}(z):=\sum_{k=0}^{\beta} f_{k, j} z^{k}$. Therefore, by (4) and the fact that the sum and the scalar multiplication are continuous operations on the topological vector space $X$, we get

$$
f_{j} \rightarrow p \quad(j \rightarrow \infty)
$$

On the other hand,

$$
\left(\sum_{n=1}^{N_{j}} \lambda_{j, n} T_{n}\right) f_{j}(z)=\left(\sum_{n=1}^{N_{j}} \lambda_{j, n} T_{n}\right)\left(\sum_{k=0}^{\beta} f_{k, j} z^{k}\right)=\sum_{k=0}^{\beta} s(j, k) f_{k, j} z^{k}
$$

So, by (5) and again by the fact that $X$ is a topological vector space,

$$
\left(\sum_{n=1}^{N_{j}} \lambda_{j, n} T_{n}\right) f_{j} \rightarrow q \quad(j \rightarrow \infty)
$$

Finally, (6) and (7) tell us that the closure of the set $S$ contains the set of all pairs $(p, q)$ ( $p, q$ polynomials; $p$ with all its coefficients nonzero). But the set of such polynomials $p$ is dense in $\mathcal{P}$, because if $P(z):=\sum_{k=0}^{m} c_{k} z^{k} \in \mathcal{P}$ and $A:=\left\{k \in\{0, \ldots, m\}: c_{k}=0\right\}$ then the sequence $P_{n}(z):=\sum_{k=0}^{m} c_{k, n} z^{k}$ $(n \in \mathbb{N})$ given by $c_{k, n}=\left\{\begin{array}{lll}c_{k} & \text { if } & k \notin A \\ 1 / n & \text { if } & k \in A\end{array}\right.$ satisfies $P_{n} \rightarrow P(n \rightarrow \infty)$ in $X$ (still one more we have used that the sum and the multiplication by scalars are continuous operations on $X$ ) and every coefficient of every $P_{n}$ is nonzero. Thus, the closure of $S$ contains $\mathcal{P} \times \mathcal{P}$, which is dense in $X \times X$ because $X$ is a CP-space. Consequently, $S$ is dense in $X \times X$, as required.

We can give similarly a characterization of the other kinds of cyclicity of a sequence of coefficient multipliers. The proof is analogous, so it is left to the reader.

Theorem 3.3. Under the same notations and conditions of Theorem 3.2, we have that the following conditions are equivalent:

(a) The set $S C\left(\left(T_{n}\right)\right)\left(H C\left(\left(T_{n}\right)\right)\right)$ is residual in $X$.

(b) The sequence $\left(T_{n}\right)$ is supercyclic (hypercyclic, resp.).

(c) The set $\left\{\lambda\left(a_{k, n}\right)_{k \geq 0}: \lambda \in \mathbb{C}, n \in \mathbb{N}\right\} \quad\left(\left\{\left(a_{k, n}\right)_{k \geq 0}: n \in \mathbb{N}\right\}\right.$, resp. $)$ is dense in $\mathbb{C}^{\mathbb{N}_{0}}$. 
Thanks to Theorem 3.3 we will be able to establish the non-supercyclicity, so the non-hypercyclicity, of every single coefficient multiplier $T_{\sigma}$.

Corollary 3.4. Assume that $T_{\sigma}$ is a coefficient multiplier on a CP-space defined on some domain $G \subset \mathbb{C}$ with $0 \in G$. Then $T_{\sigma}$ is not supercyclic, so it is not hypercyclic.

Proof. Assume, by way of contradiction, that $T_{\sigma}$ is supercyclic. Let $\sigma=$ $\left(a_{k}\right)_{k \geq 0}$. From Theorem 3.3(c), we have that the set $\left\{\lambda\left(a_{0}^{n}, a_{1}^{n}\right): \lambda \in\right.$ $\mathbb{C}, n \in \mathbb{N}\}$ must be dense in $\mathbb{C}^{2}$. But this would imply that the operator $A:(z, w) \in \mathbb{C}^{2} \mapsto\left(a_{0} z, a_{1} w\right) \in \mathbb{C}^{2}$ is supercyclic, which is absurd because no finite-dimensional space with dimension strictly larger than 1 supports a supercyclic operator (see [15]).

Notice that in the case of a sequence $\left(T_{n}\right)$ of coefficient multipliers we can get examples of hypercyclicity, even if we look for special cases, like Euler differential operators or Hadamard operators.

Proposition 3.5. (a) In every CP-space there exists a hypercyclic sequence of coefficient multipliers.

(b) If $G$ is a simply connected domain in $\mathbb{C}$ with $0 \in G$ then there exist a hypercyclic sequence of Hadamard operators and a hypercyclic sequence of Euler differential operators on $H(G)$.

Proof. (a) Assume that $X$ is a CP-space on some domain $G \subset \mathbb{C}$ containing the origin and that $\sigma=\left(a_{k}\right)_{k \geq 0} \in \mathbb{C}^{\mathbb{N}_{0}}$ is an almost null sequence, that is, there exists $N \in \mathbb{N}$ such that $a_{k}=0$ for all $k>N$. If $f \in X$ and $f(z)=\sum_{k=0}^{\infty} f_{k} z^{k}$ around the origin then $T_{\sigma} f(z)=\sum_{k=0}^{N} a_{k} f_{k} z^{k}$, so $T_{\sigma} f \in X$ because $\mathcal{P} \subset X$. In addition, we already know that each coefficient functional $f \in X \mapsto f_{k}=f^{(k)}(0) / k ! \in \mathbb{C}\left(k \in \mathbb{N}_{0}\right)$ is continuous. Since $X$ is a topological vector space we get that the mapping $f \mapsto \sum_{k=0}^{N} a_{k} f_{k} z^{k}$ is continuous or, that is the same, the coefficient multiplier $T_{\sigma}$ is a well-defined operator on $X$.

Consider now the countable set $C:=\left\{\sigma(n)=\left(a_{k, n}\right)_{k \geq 0}\right\} \subset \mathbb{C}^{\mathbb{N}_{0}}$ of all almost null sequences whose entries have rational real and imaginary parts. It is clear that $C$ is dense in $\mathbb{C}^{\mathbb{N}_{0}}$. Therefore, by Theorem 3.3, the sequence $\left(T_{\sigma(n)}\right)$ is hypercyclic on $X$. 
(b) If $C$ is the same countable set of the proof of (a) and $\sigma(n)=\left(a_{k, n}\right)_{k \geq 0} \in$ $C$ then there exists $m(n) \in \mathbb{N}$ such that $a_{k, n}=0$ for all $k>m(n)$. Now, there exists a (Lagrange interpolation) polynomial $\Phi_{n}$ such that

$$
\Phi_{n}(k)=a_{k, n} \quad(k=0, \ldots, m(n)) .
$$

But, due to the structure of the open subsets of $\mathbb{C}^{\mathbb{N}_{0}}$, the set $C_{1}:=\{s(n)=$ $\left.\left(\Phi_{n}(k)\right)_{k \geq 0}: n \in \mathbb{N}\right\}$ is also dense in $\mathbb{C}^{\mathbb{N}_{0}}$. Finally, $H(G)$ is a CP-space and, trivially, each $\Phi_{n}$ belongs to $H(\mathbb{C} \backslash\{1\})$ and it is an entire function of subexponential type, so the Hadamard operator $H_{\Phi_{n}}$ and the Euler differential operator $\Phi_{n}(\mathcal{D})$ are defined on $H(G)$, and $H_{\Phi_{n}}=T_{s(n)}=\Phi_{n}(\mathcal{D})(n \in \mathbb{N})$. Finally, $\left(T_{s(n)}\right)$ is a hypercyclic sequence due to Theorem 3.3. This concludes the proof.

In contrast to Corollary 3.4, cyclic coefficient multipliers do exist. In fact we get a characterization of such operators.

Theorem 3.6. Let $X$ be a CP-space on some domain containing the origin and let $T=T_{\sigma}$ be a coefficient multiplier on $X$. Let $\sigma=\left(a_{k}\right)_{k \geq 0}$. Then $T$ is cyclic if and only if the points $a_{k}(k \geq 0)$ are pairwise different. In this case, the set of cyclic functions for $T$ is residual in $X$.

Proof. The last part of the statement comes from Theorem 3.2 and from the facts that $T$ is cyclic if and only if $\left(T^{n}\right)_{n \geq 0}$ is cyclic and that $T^{n}=T_{\sigma(n)}$ $(n \geq 0)$, where $\sigma(n)=\left(a_{k}^{n}\right)_{k \geq 0}$.

As for the equivalence of the first part of the statement, we obtain from Theorem 3.2 that $T$ is cyclic if and only if the set

$$
S:=\operatorname{span}\left(\left\{\left(a_{k}^{n}\right)_{k \geq 0}: n \in \mathbb{N}\right\}\right)
$$

is dense in $\mathbb{C}^{\mathbb{N}_{0}}$. Observe now that $S=\left\{\left(P\left(a_{k}\right)\right)_{k \geq 0}: P \in \mathcal{P}\right\}$.

Assume that there are $p, q \in \mathbb{N}_{0}$ with $p \neq q$ and $a_{p}=a_{q}$. If $S$ were dense in $\mathbb{C}^{\mathbb{N}_{0}}$ then the set $\left\{\left(P\left(a_{p}\right), P\left(a_{q}\right)\right) \in \mathbb{C}^{2}: P \in \mathcal{P}\right\}$ would be dense in $\mathbb{C}^{2}$, which is obviously false because it lies on the diagonal of $\mathbb{C}^{2}$. Thus, $S$ is not dense.

Conversely, suppose that the points $a_{k}(k \geq 0)$ are pairwise different. It suffices to show that for given $N \in \mathbb{N}_{0}$ the set $S_{N}:=\left\{\left(P\left(a_{0}\right), \ldots, P\left(a_{N}\right)\right) \in\right.$ $\left.\mathbb{C}^{N+1}: P \in \mathcal{P}\right\}$ is dense in $\mathbb{C}^{N+1}$. In fact, $S_{N}=\mathbb{C}^{N+1}$; indeed, an interpolating polynomial $P$ for the (different) points $a_{0}, \ldots, a_{N}$ and the corresponding prefixed complex values $w_{0}, \ldots, w_{N}$ is at our disposal, and we are done. 
Remark 3.7. Theorem 3.6 could also have been derived by using [14, Proposition 3.6] -which deals with upper triangular operators- along with a "projecting onto coordinates" argument.

If $X$ is an F-space, then a sequence $\left(T_{n}\right)$ of operators on $X$ is said to satisfy the Hypercyclicity Criterion provided there exist dense subsets $X_{0}$ and $Y_{0}$ of $X$ and an increasing sequence $\left(n_{j}\right) \subset \mathbb{N}$ satisfying the following condition: $T_{n_{j}} x \rightarrow 0(j \rightarrow \infty)$ for all $x \in X_{0}$, and for any $y \in Y_{0}$ there is a sequence $\left(u_{j}\right)$ in $X$ such that $u_{j} \rightarrow 0$ and $T_{n_{j}} u_{j} \rightarrow y(j \rightarrow \infty)$. Note that this is an equivalent reformulation of the Hypercyclicity Criterion as stated in [4, Definition 1.2 and Remark 2.6], see also [10] and [2]. It gives a sufficient condition under which a sequence of operators is hypercyclic. It is still an open problem whether every hypercyclic operator satisfies the Hypercyclicity Criterion, that is, whether the sequence $\left(T^{n}\right)$ of its iterates satisfies it.

We have seen that there are hypercyclic sequences of coefficient multipliers. To finish this section, we now prove that, on the contrary, no sequence $\left(T_{n}\right)$ on a CP-space $X$ satisfies the Hypercyclicity Criterion: Assume, by way of contradiction, that $\left(T_{n}\right)$ satisfies it, and let $\left(n_{j}\right)$ be the sequence of positive integers given by such criterion. Then any subsequence $\left(m_{j}\right)$ of $\left(n_{j}\right)$ also satisfies the Hypercyclicity Criterion, whence $\left(T_{m_{j}}\right)$ is also hypercyclic. Let $T_{n}=T_{\sigma(n)}$ with $\sigma(n)=\left(a_{k, n}\right)_{k \geq 0}$, so that if $f \in X$ and $f(z)=\sum_{k=0}^{\infty} f_{k} z^{k}$ around the origin then $T_{n} f(z)=\sum_{k=0}^{\infty} a_{k, n} f_{k} z^{k}$ around the origin. If $f \in H C\left(\left(T_{n_{j}}\right)\right)$ then the sequence $\left(a_{k, n_{j}} f_{k}\right)_{j \geq 0}$ must be dense in $\mathbb{C}$ for all $k \geq 0$ (and $f_{k} \neq 0$ ) because convergence in $X$ implies convergence of the Taylor coefficients at the origin. In particular, $\left(a_{0, n_{j}}\right)_{j \geq 0}$ is dense in $\mathbb{C}$. Therefore there exists an increasing subsequence $\left(m_{j}\right)$ of $\left(n_{j}\right)$ with $a_{0, m_{j}} \rightarrow 0$ $(j \rightarrow \infty)$. But according to Theorem 3.3 this yields the non-hypercyclicity of $\left(T_{m_{j}}\right)$, that is absurd.

\section{Linear structure}

In order to conceive more specifically how big the set $C(T)$ of cyclic vectors of a cyclic coefficient multiplier $T$ can be, we establish in Theorem 4.2 that if $f$ is cyclic for $T$ then "many" functions in the span of its orbit $\left\{T^{n} f: n \geq 0\right\}$ are also cyclic. This statement can be improved if, in addition, $X$ is Banach.

In the setting of Banach spaces we will need some background about Dunford's functional calculus and general spectral theory (see, for instance, 
[6, Chapter 1] or [23, Chapter 10]). If $L$ is an operator on a complex Banach space $E$ then $\sigma(L)$ will stand for its spectrum, that is, $\sigma(L)=\{\lambda \in \mathbb{C}: L-\lambda I$ is not invertible\}. If $L^{*}$ is the adjoint of $L$, then $\sigma(L)=\sigma\left(L^{*}\right)$. The point spectrum $\sigma_{p}(L)$ of $L$ is the set of eigenvalues of $L$, that is, the set of $\lambda \in \mathbb{C}$ such that $L-\lambda I$ is not one-to-one $\left(\right.$ so $\left.\sigma_{p}(L) \subset \sigma(L)\right)$. Denote by $\mathcal{F}(L)$ the family of all functions $\Phi$ which are holomorphic on some domain $D(\Phi)$ containing $\sigma(L)$. Hence $H(\mathbb{C}) \subset \mathcal{F}(L)=\mathcal{F}\left(L^{*}\right)$. Let $\Phi \in \mathcal{F}(L)$ and $\gamma$ be a positively oriented Jordan cycle surrounding counterclockwise $\sigma(L)$ such that both $\gamma$ and its geometric interior are contained in $D(\Phi)$. Then the operator $\Phi(L)$ is defined by the following equation, where the integral exists as a limit of Riemann sums in the norm of the space of operators on $E$ :

$$
\Phi(L)=\frac{1}{2 \pi i} \oint_{\gamma} \Phi(\lambda)(\lambda I-T)^{-1} d \lambda .
$$

Then $\Phi(L)$ depends only of $\Phi$, and the notion of $\Phi(L)$ extends the definition $P(L)=\sum_{j=0}^{N} a_{j} L^{j}$ when $P(z)$ is the polynomial $P(z)=\sum_{j=0}^{N} a_{j} z^{j}$. Another important property is that $\Phi\left(L^{*}\right)=\Phi(L)^{*}$.

We will make use of the following result, which might be of some interest in itself.

Lemma 4.1. Let $T=T_{\sigma}$ be a coefficient multiplier defined on a Banach $C P$-space $X$ by a complex sequence $\sigma$. Then

$$
\sigma_{p}\left(T^{*}\right)=\sigma
$$

In particular, $\sigma$ is bounded.

Proof. We have that $X$ is a $\mathrm{CP}$-space on some domain $G \subset \mathbb{C}$ with $0 \in$ $G$. The last sentence of the statement derives from the inclusion $\sigma_{p}\left(T^{*}\right) \subset$ $\sigma\left(T^{*}\right)=\sigma(T)$ and from the compactness of the spectrum of an operator on a Banach space.

As for the first part, suppose that $\sigma=\left(a_{k}\right)_{k \geq 0}$. Fix $k \in \mathbb{N}_{0}$ and consider the linear functional $\varphi \in X^{*}(:=$ the topological dual space of $X)$ defined as follows: If $f \in X$ and $f(z)=\sum_{j=0}^{\infty} f_{j} z^{j}$ around the origin, then $\varphi(f)=f_{k}$. Note that the continuity of $\varphi$ is a consequence of the fact that $X$ is a CPspace. Then $\varphi \neq 0$. On the other hand, $\left(T^{*} \varphi\right)(f)=\varphi(T f)=a_{k} f_{k}=a_{k} \varphi(f)$ for all $f \in X$, so $T^{*} \varphi=a_{k} \varphi$. Consequently, $\varphi$ is an eigenvector for $T^{*}$ with eigenvalue $a_{k}$. Thus, $a_{k} \in \sigma_{p}\left(T^{*}\right)\left(k \in \mathbb{N}_{0}\right)$ and $\sigma \subset \sigma_{p}\left(T^{*}\right)$. 
Conversely, assume that $\lambda \in \sigma_{p}\left(T^{*}\right)$. Then there must be a functional $\varphi \in X^{*} \backslash\{0\}$ with $T^{*} \varphi=\lambda \varphi$. By linearity and the denseness of $\mathcal{P}$ in $X$, we can find $m \in \mathbb{N}_{0}$ such that $\varphi\left(h_{m}\right) \neq 0$, where $h_{m}(z):=z^{m}$. But $\left(T^{*} \varphi\right)\left(h_{m}\right)=$ $\lambda \varphi\left(h_{m}\right)$, so $\varphi\left(T h_{m}\right)=\lambda \varphi\left(h_{m}\right)$. Now, $\varphi\left(T h_{m}\right)=\varphi\left(a_{m} h_{m}\right)=a_{m} \varphi\left(h_{m}\right)$, whence $a_{m} \varphi\left(h_{m}\right)=\lambda \varphi\left(h_{m}\right)$. Since $\varphi\left(h_{m}\right) \neq 0$, we obtain $\lambda=a_{m} \in \sigma$, which yields $\sigma_{p}\left(T^{*}\right) \subset \sigma$, as desired.

We remark that due to the last lemma any function $\Phi \in \mathcal{F}\left(T_{\sigma}\right)$ makes sense on the points of $\sigma$.

Theorem 4.2. Assume that $X$ is a CP-space on some domain containing the origin and that $f \in C\left(T_{\sigma}\right)$, where $T_{\sigma}$ is a coefficient multiplier defined on $X$ by a sequence $\sigma=\left(a_{k}\right)_{k \geq 0}$. Let us set $S:=\operatorname{span}\left(\left\{T_{\sigma}^{n} f: n \geq 0\right\}\right)$ and $A:=\left\{P\left(T_{\sigma}\right) f: P \in \mathcal{P}, P\left(a_{k}\right) \neq 0\right.$ for all $\left.k \in \mathbb{N}_{0}\right\}$. We have the following:

(a) The set $A$ is a subset of $S$ which is dense in $S$, hence in $X$.

(b) $A \subset C\left(T_{\sigma}\right)$.

(c) If $X$ is a Banach space and

$$
B:=\left\{\Phi\left(T_{\sigma}\right) f: \Phi \in \mathcal{F}\left(T_{\sigma}\right), \Phi\left(a_{k}\right) \neq 0 \text { for all } k \in \mathbb{N}_{0}\right\},
$$

then $A \subset B \subset C\left(T_{\sigma}\right)$. In particular, $B$ is dense in $X$.

Proof. For the sake of simplicity, we denote $T=T_{\sigma}$. Note first that, since $T$ is cyclic and $f \in C(T)$, the points $a_{k}\left(k \in \mathbb{N}_{0}\right)$ are pairwise different and the set $S$ is dense in $X$.

(a) Observe that $S=\{P(T) f: P \in \mathcal{P}\}$, whence $A \subset S$. As for the density, fix a function $g \in S \backslash A$ and a neighbourhood $U$ of $g$ in $X$. Then $g=P(T) f$ for some $P \in \mathcal{P}$ with some zero in $\left\{a_{k}: k \geq 0\right\}$. We can suppose that $g \not \equiv 0$, for in this case a multiple $\lambda f$ of $f$, where $\lambda$ is an adequate nonzero small constant, satisfies $\lambda f \in A \cap U$. Therefore $P(z)=$ $\gamma \prod_{j=1}^{r}\left(z-\alpha_{j}\right)^{m(j)} \cdot \prod_{j=1}^{s}\left(z-\beta_{j}\right)^{n(j)}$ for certain $r \in \mathbb{N}, s \in \mathbb{N}, \gamma \in \mathbb{C} \backslash\{0\}$, $\alpha_{j} \in\left\{a_{k}: k \geq 0\right\}(j=1, \ldots, r), \beta_{j} \in \mathbb{C} \backslash\left\{a_{k}: k \geq 0\right\}(j=1, \ldots, s)$, $m(j) \in \mathbb{N}(j=1, \ldots, r), n(j) \in \mathbb{N}_{0}(j=1, \ldots, s)$. Since the coefficients of a polynomial depend continuously on its roots and $\mathbb{C} \backslash\left\{a_{k}\right\}_{k \geq 0}$ is dense in $\mathbb{C}$, we can move slightly $\alpha_{1}, \ldots \alpha_{r}$ to respectively close points $\alpha_{1}^{\prime}, \ldots \alpha_{r}^{\prime}$ which are not in $\left\{a_{k}\right\}_{k \geq 0}$, in such a way that $P_{1}(T) f \in U$ (we have used again that $X$ is a topological vector space), where $P_{1}(z)$ has the same expression 
as $P(z)$ except that the points $\alpha_{j}$ are replaced to $\alpha_{j}^{\prime}(j=1, \ldots, r)$. So $P_{1}(T) f \in A \cap U$, which shows the density of $A$ in $S$.

(b) In order to prove that $A \subset C(T)$, we fix $g=Q(T) f \in A$. Then $Q \in \mathcal{P}$ and $Q\left(a_{k}\right) \neq 0$ for all $k \in \mathbb{N}_{0}$. We should show that $\operatorname{span}\left(\left\{T^{n} g: n \geq 0\right\}\right)$ is dense in $X$. But

$$
\begin{aligned}
\operatorname{span}\left(\left\{T^{n} g: n \geq 0\right\}\right) & =\{P(T) Q(T) f: P \in \mathcal{P}\} \\
& =Q(T)(\{P(T) f: P \in \mathcal{P}\}) \\
& =Q(T)(S) .
\end{aligned}
$$

We now recall that $S$ is dense in $X$. Thus, the proof of this part will be finished as soon as we show that the operator $Q(T)$ has dense range. For this, observe that $Q(T)$ has a compositional factorization into finitely many operators of the form $\mu I(\mu \in \mathbb{C} \backslash\{0\}), T-\lambda I\left(\lambda \in \mathbb{C} \backslash\left\{a_{k}: k \geq 0\right\}\right)$. An operator $\mu I$ has obviously dense range, so we have only to prove that $T-\lambda I$ has dense range. Since $X$ is a CP-space, it is enough to see that $(T-\lambda I)(X) \supset \mathcal{P}$. By linearity, it is in turn enough to show that each monomial $z^{m}\left(m \in \mathbb{N}_{0}\right)$ is in $(T-\lambda I)(X)$. This is easy, because the function $F(z):=z^{m} /\left(a_{m}-\lambda\right)$ is in $X$ (recall that $\mathcal{P} \subset X$ and that $a_{m}-\lambda \neq 0$ ) and $(T-\lambda I) F(z)=z^{m}$.

(c) Since $\mathcal{P} \subset H(\mathbb{C})$ we have that, trivially, $A \subset B$. Therefore our goal is to show that every member of $B$ is a cyclic function for $T$. Consider a function $g \in B$. Then there is $\Phi \in \mathcal{F}(T)$ such that $g=\Phi(T) f, \Phi$ is holomorphic on a domain $D(\Phi) \supset \sigma(T)$ and $\Phi\left(a_{k}\right) \neq 0$ for all $k \in \mathbb{N}_{0}$. At this point we distinguish two cases.

If $\Phi$ is constant, say $\Phi(z) \equiv \lambda \neq 0$, then $g=\lambda f$. Hence $g$ is obviously cyclic because $f$ is.

If $\Phi$ is nonconstant, then a refinement of the spectral mapping theorem [23, Theorem 10.33] asserts that $\sigma_{p}\left(\Phi\left(T^{*}\right)\right)=\Phi\left(\sigma_{p}\left(T^{*}\right)\right)$. By Lemma 4.1, we get $\sigma_{p}\left(\Phi\left(T^{*}\right)\right)=\Phi\left(\left\{a_{k}: k \in \mathbb{N}_{0}\right\}\right)$. Thus, $0 \notin \sigma_{p}\left(\Phi\left(T^{*}\right)\right)$. But $\Phi\left(T^{*}\right)=$ $\Phi(T)^{*}$, whence $0 \notin \sigma_{p}\left(\Phi(T)^{*}\right)$. Now, a direct application of the Hahn-Banach theorem drives us to assert that $\Phi(T)$ has dense range. Finally, similarly to the proof of (b), we have

$$
\operatorname{span}\left(\left\{T^{n} g: n \in \mathbb{N}_{0}\right\}\right)=\{P(T) \Phi(T) f: P \in \mathcal{P}\}=\Phi(T)(S) .
$$

Consequently, the last span is dense in $X$ because $S$ is dense and $\Phi(T)$ has dense range. In other words, $g \in C(T)$, as required. 
We have shown in Theorem 3.2 that the set of cyclic functions of a cyclic sequence $\left(T_{n}\right)$ of coefficient multipliers is in fact residual; in less precise words, such set is large in a topological sense. On the other hand, it is evident that a sum $f+g$ of two cyclic functions $f, g$ is in general noncyclic (take $g=-f$ ), so $C\left(\left(T_{n}\right)\right)$ is not a linear manifold. Of course, if $\lambda \in \mathbb{C} \backslash\{0\}$ and $f \in C\left(\left(T_{n}\right)\right)$ then $\lambda f \in C\left(\left(T_{n}\right)\right)$. Then a natural question arises: Is $C\left(\left(T_{n}\right)\right)$ large in an algebraic sense, that is, is there a "large" manifold $M \subset X$ such that $M \backslash\{0\} \subset C\left(\left(T_{n}\right)\right)$ ? The existence of such "large" -in several ways: dense, infinite-dimensional closed, etc- manifolds has appeared in the literature for hypercyclic and supercyclic operators or sequences of operators (see the surveys [10], [13], [20]). As for the coefficient multipliers, the definitive answer is a little disappointing, as stated in the next proposition, which puts the end on this paper.

Proposition 4.3. Let $\left(T_{n}\right)$ be a cyclic sequence of coefficient multipliers defined on a CP-space $X$, and $M \neq\{0\}$ be a linear submanifold such that $M \backslash\{0\} \subset C\left(\left(T_{n}\right)\right)$. Then $\operatorname{dim} M=1$.

Proof. Assume, by way of contradiction, that $\operatorname{dim} M \geq 2$. Then there are $f, g \in M$ such that $f+\lambda g \not \equiv 0$ and $f+\lambda g \in C\left(\left(T_{n}\right)\right)$ for all $\lambda \in \mathbb{C}$. Let $f(z)=\sum_{k=0}^{\infty} f_{k} z^{k}, g(z)=\sum_{k=0}^{\infty} g_{k} z^{k}$ around the origin. Then, as in the proof of Theorem 3.2, $f_{k} \neq 0 \neq g_{k}\left(k \in \mathbb{N}_{0}\right)$. In particular, $f_{0} \neq 0 \neq g_{0}$. Define $\lambda:=-f_{0} / g_{0}$ and $h:=f+\lambda g$. Then $h \in M \backslash\{0\}$ but $h \notin C\left(\left(T_{n}\right)\right)$ because $h_{0}=0$. This is the desired contradiction.

\section{References}

[1] S. Axler, P. Bourdon, W. Ramey, Harmonic function theory, SpringerVerlag, (New York, 1992).

[2] T. Bermúdez, A. Bonilla, A. Peris, On Hypercyclicity and Supercyclicity Criterion, Bull. Austral. Math. Soc., 70 (2004), 45-54.

[3] L. Bernal-González, Universal functions for Taylor shifts, Complex Variables, 31 (1996), 121-129.

[4] J. Bès, A. Peris, Hereditarily hypercyclic operators, J. Funct. Anal., 167 (1999), 94-112. 
[5] L. Bieberbach, Analytische Fortsetzung, Springer, (Berlin, 1955).

[6] H.R. Dowson, Spectral Theory of Linear Operators, Academic Press, (London, 1978).

[7] P.L. Duren, Theory of $H^{p}$ spaces, Academic Press, (New York, 1970).

[8] L. Frerick, Coefficient multipliers with closed range, Note di Mat., 17 (1997), 61-70.

[9] G. Godefroy and J.H. Shapiro, Operators with dense, invariant, cyclic vector manifolds, J. Funct. Anal., 98 (1991), 229-269.

[10] K.-G. Große-Erdmann, Universal families and hypercyclic operators, Bull. Amer. Math. Soc. (N.S.), 36 (1999), 345-381.

[11] K.-G. Große-Erdmann, Hypercyclic and chaotic weighted shifts, Studia Math., 139 (2000), 47-68.

[12] K.-G. Große-Erdmann, Rate of growth of hypercyclic entire functions, Indag. Math., 11 (2000), 561-571.

[13] K.-G. Große-Erdmann, Recent developments in Hypercyclicity, Rev. R. Acad. Cien. Serie A. Mat., 97 (2003), 273-286.

[14] D. Herrero, D. Larson and W. Wogen, Semitriangular operators, Houston J. Math., 17 (1991), 477-499.

[15] G. Herzog, On linear operators having supercyclic vectors, Studia Math., 103 (1992), 295-298.

[16] E. Hille, Analytic Function Theory, vol. II, Chelsea Publishing Company, (New York, 1987).

[17] F. Martínez-Giménez, A. Peris, Chaos for backward shift operators, J. Bifur. Chaos Appl. Sci. Engrg., 12 (2002), 1703-1715.

[18] V. Mathew, A note on hypercyclic operators on the space of entire sequences, Indian J. Pure and Appl. Math., 25 (1994), 1181-1184.

[19] A. Montes, H. Salas, Supercyclic subspaces: spectral theory and weighted shifts, Adv. Math., 163 (2001), 74-134. 
[20] A. Montes, H. Salas, Supercyclic subspaces, Bull. London Math. Soc., 35 (2003), 721-737.

[21] J. Müller, The Hadamard multiplication theorem and applications in summability theory, Complex Variables, 18 (1992), 155-166.

[22] S. Rolewicz, On orbits of elements, Studia Math., 32 (1969), 17-22.

[23] W. Rudin, Functional Analysis, McGraw-Hill, (New York, 1991).

[24] A.L. Shields, Weighted shift operators and analytic function theory, in Topics of Operator Theory, C. Pearcy (ed.), American Mathematical Society, (Providence, Rhode Island, 1974).

[25] K. Zhu, Operator Theory in Functions Spaces, Marcel-Dekker, (New York, 1990).

L. Bernal-González, M.C. Calderón-Moreno and J.A. Prado-Bassas Departamento de Análisis Matemático

Universidad de Sevilla, Apdo. 1160

Avda. Reina Mercedes 41080-Sevilla, Spain

E-mails: lbernal@us.es, mccm@us.es, bassas@us.es 